\title{
ATIVIDADE ANTIARRITMOGENICA DA LEVOMEPROMAZINA EM CÃES SUBMETIDOS À ANESTESIA PELA QUETAMINA
}

\author{
THE USE OF LEVOMEPROMAZINE TO BLOCKADE THE ARRHYTHMIA INDUCED \\ BY THE EPINEPHRINE IN DOGS ANESTHETIZED WITH KETAMINE
}

\author{
Newton Nunes ${ }^{1}$ Flávio Massone ${ }^{2}$ Luiz Gonzaga Pompermayer ${ }^{3}$ \\ Josmari Pirolo $^{4}$ Aparecido Antonio Camacho ${ }^{5}$
}

RESUMO

Este experimento teve por objetivo avaliar a viabilidade do emprego da levomepromazina no bloqueio da atividade arritmogênica da adrenalina, em cães anestesiados pela quetamina. Para tal, foram utilizados 30 cães adultos, machos e fêmeas, considerados sadios, com pesos compreendidos entre $7 e$ $14 \mathrm{~kg}$. Estes foram divididos em 3 grupos de 10 animais $(G 1, G 2$ e G3). Aos cães de G1 foi administrada, por via intravenosa, adrenalina em doses de 3, 6, 9, 12 e $15 \mu \mathrm{g} / \mathrm{kg}$, em intervalos de 10 minutos. Deste grupo, foram colhidos o tempo de duração do efeito da catecolamina (TA), estabelecido pela contagem da freqüência cardíaca e o número total de batimentos cardíacos de origem ectópica, produzidos pela adrenalina (ESV). Aos animais do G2, foi administrada solução salina a 0,9\%, na dose de $0,2 \mathrm{ml} / \mathrm{kg}$, por via intravenosa, seguida, 10 minutos após, da injeção, pela mesma via, de quetamina, na dose de $2 \mathrm{mg} / \mathrm{kg}$. Decorridos 5 minutos, iniciou-se a infusão contínua de quetamina, por via intravenosa, na dose de $0,2 \mathrm{mg} / \mathrm{kg} / \mathrm{min}$. Aguardou-se 5 minutos e iniciou-se a administração de adrenalina e colheita das variáveis, conforme protocolado para o G1. Aos animais do G3, aplicou-se a mesma metodologia, substituindo-se o placebo pela levomepromazina, administrada por via intravenosa, na dose de $1 \mathrm{mg} / \mathrm{kg}$. A análise dos resultados mostrou que a levomepromazina reduz a duração do efeito da catecolamina e minimiza o aparecimento de batimentos cardíacos de origem ectópica. Os achados permitiram concluir que a levomepromazina é útil no bloqueio da arritmia produzida pela adrenalina em cães anestesiados pela quetamina.

Palavras-chave: levomepromazina, quetamina, arritmia, cães.

\section{SUMMARY}

The use of levomepromazine to blockade the arrhythmia produced by the use of epinephrine in dogs given ketamine was evaluated. Thirthy adults crossbreed dogs, males and females with average weight from 7 to $15 \mathrm{~kg}$ were used. The animals were alocated in three groups of 10 dogs each $(G 1, G 2$ and G3). To the G1 was administered epinephrine at 3, 6, 9, 12 and $15 \mu \mathrm{g} / \mathrm{kg}$, intravenously, at 10 minutes intervals. From this dogs was measured the time of action of epinephrine (TA) and the number of premature ventriculary complexes (ESV), obtainded. To the G2 was intravenously administered $0.2 \mathrm{ml} / \mathrm{kg}$ of saline solution. Ten minutes after each dog received $2 \mathrm{mg} / \mathrm{kg}$ of ketamine and five minutes after it was started the intravenous continuously administered ketamine at $0.2 \mathrm{mg} / \mathrm{kg} / \mathrm{min}$. After five minutes the epinephrine was given as the same way, doses and intervals as described for G1, followed by the measurements of TA and ESV. To the dogs from G3 was used the same method but replacing the saline solution by levomepromazine intravenously administered at $1 \mathrm{mg} / \mathrm{kg}$. The results showed that the levomepromazine decrease the TA and reduce the ESV, in ketamine anesthetized dogs.

Key words: levomepromazine, ketamine, arrhythmia, dogs.

\section{INTRODUÇÃO}

Desde os primórdios da anestesiologia, tem-se tentado associar diversos fármacos, para se obter anestesia adequada ao procedimento cirúrgico

\footnotetext{
${ }^{1}$ Professor Assistente, Doutor, Departamento de Clínica e Cirurgia Veterinária. Faculdade de Ciências Agrárias e Veterinárias FCAV/UNESP - Campus de Jaboticabal, SP, 14870-000. E-mail: newton@ fcav.unesp.br. Autor para correspondência.

${ }^{2}$ Professor Titular, Departamento de Cirurgia e Reprodução Animal, Faculdade de Medicina Veterinária e Zootecnia FMVZ/UNESP Campus de Botucatu, SP.

${ }^{3}$ Professor Adjunto, Departamento de Veterinária. Universidade Federal de Viçosa, Viçosa, MG.

${ }^{4}$ Professor Adjunto, Departamento de Clínicas Veterinárias. Universidade Estadual de Londrina, Londrina, PR.

${ }^{5}$ Professor Adjunto, Departamento de Clínica e Cirurgia Veterinária, FCAV/UNESP.
} 
proposto, aliada à segurança e praticidade no seu emprego. Com o advento de novas técnicas e com a introdução de drogas dotadas de diferentes características, o arsenal de que o profissional dispõe faz com que o anestesiologista tenha uma ampla gama de opções.

Entre os fármacos de uso rotineiro em Anestesiologia Veterinária, os fenotiazínicos têm se destacado, principalmente devido ao efeito tranqüilizante e à potencialização dos agentes anestésicos dissociativos ou barbitúricos, obtidos com seu emprego, aliados a outros fatores, de não menor importância, como por exemplo, as ações antiemética, anti-histamínica e, principalmente, adrenolítica.

Os derivados fenotiazínicos produzem sonolência e hipotonia muscular com diminuição dos reflexos motores. Causam depressão do sistema nervoso central por sua ação sobre os centros nervosos inferiores, tálamo, hipotálamo e formação reticular, além de possuírem propriedades antieméticas, anti-histamínicas e antiespasmódicas (SHORT, 1987).

Sobre a atividade cardiovascular, os agentes do grupo determinam bloqueio $\alpha$ adrenérgico, dependente da dose, com diminuição da regulação vasomotora, levando à hipotensão (NUNES et al., 1995) e taquicardia, possuindo, entretanto, características antiarrítmicas (SHORT, 1987). Relativamente às arritmias, tem sido descrita a utilidade dos agentes $\alpha$-bloqueadores, pois as drogas deste grupo protegem contra irregularidades cardíacas induzidas pela adrenalina durante a anestesia. Tais efeitos poderiam ser explicados pela prevenção da elevação da pressão arterial, promovida pela catecolamina (HOFFMAN \& LEFKOWITZ, 1991), mecanismo que, em parte, atua no desenvolvimento de complexos prematuros ventriculares (LEMKE et al., 1994).

Dentre os fenotiazínicos, encontra-se a levomepromazina, de uso consagrado em psiquiatria, porém pouco empregada na prática clínico-cirúrgica veterinária e pouco estudada com relação às características do fármaco, em associação com demais drogas do arsenal à disposição do anestesista. A levomepromazina produz discreta analgesia e é dotada de efeito termolítico (MASSONE, 1994), oriundo da depressão dos centros termorreguladores do hipotálamo e da vasodilatação periférica, causadora de maior troca de calor com o meio ambiente (NUNES $\boldsymbol{e t} \boldsymbol{a l} ., 1995)$. Não são conhecidos estudos relativos ao potencial antiarritmogênico do fármaco, em associação à quetamina.

O cloridrato de quetamina é um derivado da fenciclidina, que atua por mecanismos de inibição do sistema corticotalâmico e ativador concomitante do sistema límbico (PENDER, 1971). Produz anestesia do tipo dissociativa de curta duração, caracterizada por ausência de respostas aos estímulos nociceptivos e manutenção dos reflexos protetores (MASSONE, 1994).

Sua ação estimulatória sobre os receptores $\alpha$-adrenérgicos e a inibição parassimpática do coração (MASSONE, 1994; PENDER, 1971; SHORT, 1987), diminuição da resposta pelos barorreceptores e interferência na recaptação da noradrenalina e demais catecolaminas pela terminação simpática foram responsabilizadas pelo aumento de freqüência e débito cardíacos.

Alguns autores relataram que o fármaco sensibiliza o miocárdio ao efeito arritmogênico da adrenalina (BEDNARSKI $\boldsymbol{e t} \boldsymbol{a l}$., 1988), embora a ação da quetamina sobre a arritmia induzida pela adrenalina, em cães, fosse estudada por NIIYA (1990), ocasião em que foi demonstrada a ausência de arritmogenicidade, quando se empregava o agente dissociativo. Nesta ocasião, o efeito antiarrítmico obtido foi imputado à capacidade depressora cardiovascular da quetamina.

Relativamente às arritmias, tem sido empregada a adrenalina, como droga experimental, na indução de alterações do ritmo e frequiência do coração. Trata-se de catecolamina produzida a partir da medula supra-renal como resposta fisiológica ao estresse. Sua ação pode determinar diminuição da resistência periférica total, vasoconstricção venosa, aumento da frequiência cardíaca, quando usada em pequenas doses, elevação do débito cardíaco, vasodilatação coronariana e aumento da pressão arterial (WYLLIE \& CHURCHILL-DAVIDSON, 1974).

A aplicação do fármaco, na forma sintética de cloridrato, por via intravenosa, determina arritmia de grau variável e dependente da dose, podendo, em casos extremos, causar síncope branca, mormente se empregada durante anestesia geral volátil por agente halogenado (MASSONE, 1994). Existem relatos de ocorrência de taquicardia, complexos prematuros ventriculares e fibrilação ventricular decorrentes de liberação endógena da catecolamina (LEMKE $\boldsymbol{e t} \boldsymbol{a l}$. , 1994).

A adrenalina tem sido empregada, ocasionalmente, na verificação da arritmogenicidade de vários agentes anestésicos, sendo que TRANQUILLI et al. (1986) definiram a dose arritmogênica da catecolamina como sendo a necessária à produção de quatro ou mais contrações prematuras ventriculares, intermitentes ou contínuas, num intervalo de 15 segundos.

Neste experimento, objetivou-se estudar a capacidade da levomepromazina em reduzir a arritmogenicidade da adrenalina em cães submetidos à anestesia pela quetamina. 


\section{MATERIAL E MÉTODOS}

Utilizaram-se trinta cães, sem raça definida, de ambos os sexos, com pesos compreendidos entre 7 e $14 \mathrm{~kg}$, adultos, considerados sadios, evitando-se fêmeas prenhes ou em estro, provenientes do canil da Faculdade de Medicina Veterinária e Zootecnia da UNESP - Câmpus de Botucatu, SP. Estes, após protocolados, foram separados, aleatoriamente, em três grupos de 10 animais (G1, G2 e G3)

Para o grupo 1 (G1), empregou-se a adrenalina $^{\mathrm{a}}$, por via intravenosa, em doses crescentes de $3 \mu \mathrm{g} / \mathrm{kg}, \quad 6 \mu \mathrm{g} / \mathrm{kg}, 9 \mu \mathrm{g} / \mathrm{kg} 12 \mu \mathrm{g} / \mathrm{kg}$ e $15 \mu \mathrm{g} / \mathrm{kg}$, em intervalos de 10 minutos. Deste grupo, foram colhidos os valores referentes à duração do efeito da catecolamina (TA), bem como o número total de batimentos cardíacos de origem ectópica (ESV) que viessem a ser observados com cada dose.

Para a avaliação do TA, acompanhou-se a freqüência cardíaca em monitor ${ }^{\mathrm{b}}$ e levou-se em consideração o período compreendido entre a aplicação do fármaco e o momento em que esta atingia $90 \%$ do valor lido imediatamente antes de sua administração. Consideraram-se, para a contagem de ESV, os complexos prematuros ventriculares direitos e esquerdos, fusões e despolarizações ectópicas de origem no nódulo atrio-ventricular, observados por meio de eletrocardiógrafo ${ }^{c}$ ajustado para registro da derivação DII, após a administração de cada dose, sendo o período de monitoramento coincidente com o TA.

Os animais do $\mathrm{G} 2$ receberam, por via intravenosa, solução salina a $0,9 \%$, em volume correspondente a $0,2 \mathrm{ml} / \mathrm{kg}$ (placebo). Decorridos $10 \mathrm{mi}-$ nutos, aplicaram-se $2 \mathrm{mg} / \mathrm{kg}$ de quetamina ${ }^{\mathrm{d}}$, por via intravenosa. Após 5 minutos, cada animal foi submetido à infusão intravenosa contínua de quetamina na dose de $0,2 \mathrm{mg} / \mathrm{kg} / \mathrm{min}$, diluídos em solução fisiológica e aplicados através de bomba de infusão ${ }^{\mathrm{e}}$, até o final do experimento.

Iniciada a infusão, aguardaramse 5 minutos e iniciou-se a aplicação de adrenalina, em doses crescentes, a intervalos de 10 minutos, bem como a colheita de TA e ESV, como fora protocolado para o G1. Para o G3, obedeceu-se a mesma metodologia de G2, substituindo-se o placebo pela levomepromazina ${ }^{f}$, na dose de $1 \mathrm{mg} / \mathrm{kg}$, aplicada por via intravenosa.

A avaliação estatística das variáveis foi efetuada por meio de Análise de Perfil (CURI, 1980; MORRISON, 1967), para interpretação dos possíveis efeitos que levaram à alteração nas médias de cada variável estudada nos diversos momentos, incluindo os testes das hipóteses de: interação entre grupos e momentos, efeito de grupos, efeito de momentos, efeito de grupo em cada momento e efeito de momento dentro de cada grupo. As médias obtidas da observação de ESV foram submetidas à "transformação raiz quadrada", sendo que, para representação gráfica procedeu-se à reconversão das mesmas ao seu valor original.

Os resultados da análise estatística são apresentados no texto, considerando um nível de significância de 5\% ( $\mathrm{p}<0,05)$. Os parâmetros estudados pela Análise de Perfil foram realizados pelo Serviço de Estatística e Computação e pelo Núcleo de Processamento de Dados da FMVZ - Campus de Botucatu - UNESP.

\section{RESULTADOS E DISCUSSÃO}

Frente aos resultados obtidos, observou-se que a variável TA foi traduzida em curvas de perfis similares, porém localizadas em níveis significativamente diferentes e crescentes, ao longo dos momentos, embora a inclinação fosse menos acentuada no G3. Com a dose de $3 \mu \mathrm{g} / \mathrm{kg}$, não foram identificadas diferenças entre os grupos. A partir de $6 \mu \mathrm{g} / \mathrm{kg}$, ocorreu diferença significativa entre as médias do G3 e às de G1 e G2, que não distaram entre si (tabela 1 e figura 1 ).

A adrenalina produziu efeito observável por tempo crescente em relação à dose administrada, sendo que, em maiores doses, a catecolamina teve sua ação atenuada nos cães tratados com a levomepromazina. Tal achado sugere que a ação antiar-
Tabela 1 - Valores médios (x) da duração do efeito da adrenalina (TA), em segundos, desvios-padrões (s) e coeficientes de variação (CV) em $\%$, obtidos em cães não tratados (G1) ou tratados com quetamina (G2 e G3) e pré-tratados com levomepromazina (G3), submetidos a doses crescentes de adrenalina.

\begin{tabular}{|c|c|c|c|c|c|c|}
\hline Tratamento & & $3 \mu \mathrm{g} / \mathrm{kg}$ & $6 \mu \mathrm{g} / \mathrm{kg}$ & $9 \mu \mathrm{g} / \mathrm{kg}$ & $12 \mu \mathrm{g} / \mathrm{kg}$ & $15 \mu \mathrm{g} / \mathrm{kg}$ \\
\hline \multirow{3}{*}{ G1 } & $\mathrm{x}$ & 87,00 & 120,00 & 132,00 & 147,00 & 153,00 \\
\hline & $\mathrm{s}$ & 9,48 & 31,62 & 28,98 & 35,91 & 53,75 \\
\hline & $\mathrm{CV}$ & 10,90 & 26,35 & 21,95 & 24,43 & 35,13 \\
\hline \multirow{3}{*}{ G2 } & $\mathrm{x}$ & 105,83 & 132,33 & 145,83 & 147,50 & 151,66 \\
\hline & $\mathrm{s}$ & 27,78 & 24,34 & 36,79 & 35,19 & 39,50 \\
\hline & $\mathrm{CV}$ & 26,25 & 18,39 & 25,23 & 23,86 & 26,04 \\
\hline \multirow{3}{*}{ G3 } & $\mathrm{x}$ & 68,75 & 82,08 & 91,08 & 96,66 & 109,83 \\
\hline & $\mathrm{s}$ & 17,85 & 14,68 & 19,66 & 30,84 & 41,73 \\
\hline & $\mathrm{CV}$ & 25,96 & 17,89 & 21,59 & 31,91 & 38,00 \\
\hline
\end{tabular}




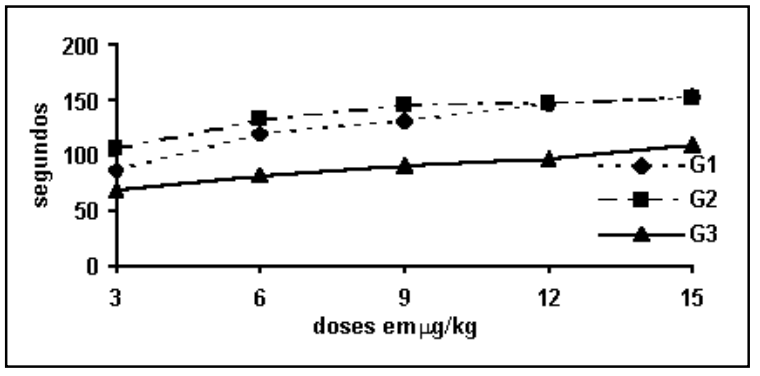

Figura 1 - Variação dos valores médios da duração do efeito da adrenalina (TA), em segundos, em cães não tratados (G1) ou tratados com quetamina (G2 e G3) e prétratados com levomepromazina (G3), submetidos a doses crescentes de adrenalina. identificada.

A levomepromazina pode ser indicada no pré-tratamento, mesmo quando se suspeita de liberação endógena excessiva de catecolaminas. Ao se observarem as curvas de ESV e a tabela 2, torna-se patente a ocorrência de batimentos ventriculares ectópicos, muitas vezes em nível inferior ao estabelecido por TRANQUILLI et al. (1986) como característico de arritmia, nos cães do G3.

A fenotiazina mostrou-se adequada no controle do desenvolvimento de focos ectópicos

rítmica da fenotiazina (SHORT, 1987) advenha da competição por receptores do tipo $\alpha_{2}$, encontrados em maior quantidade no coração.

A quetamina, empregada isoladamente, não interferiu com as médias de TA, já que não produziu valores significativamente divergentes daqueles encontrados em animais tratados apenas com adrenalina. Outrossim, pode-se sugerir que, havendo suspeita da possibilidade de desenvolverem-se arritmias advindas de liberação excessiva de catecolaminas, o prétratamento de eleição apontaria para o emprego da levomepromazina, visto que o efeito da catecolamina perduraria, nestas condições, por um menor período de tempo.

A adrenalina produziu elevada ocorrência de batimentos ventriculares de origem ectópica. O estudo de ESV mostrou que, no grupo pré-tratado com o fenotiazínico, não houve crescimento significativo da curva, ao contrário dos demais, onde os valores foram crescentes. Os cães

Tabela 2 - Valores médios transformados à raiz quadrada (x) do número total de batimentos cardíacos de origem não sinusal (ESV), desviospadrões (s) e coeficientes de variação $(C V)$ em \%, obtidos em cães não tratados (G1) ou tratados com quetamina (G2 e G3) e prétratados com levomepromazina (G3), submetidos a doses crescentes de adrenalina.

\begin{tabular}{rrrrrrr}
\hline \multirow{2}{*}{ Tratamento } & & $3 \mu \mathrm{g} / \mathrm{kg}$ & $6 \mu \mathrm{g} / \mathrm{kg}$ & $9 \mu \mathrm{g} / \mathrm{kg}$ & $12 \mu \mathrm{g} / \mathrm{kg}$ & $15 \mu \mathrm{g} / \mathrm{kg}$ \\
\hline & & & & & & \\
\hline $\mathrm{G} 1$ & $\mathrm{x}$ & 2,08 & 3,81 & 4,06 & 5,00 & 5,44 \\
& $\mathrm{~S}$ & 1,51 & 2,92 & 3,39 & 3,02 & 3,63 \\
& $\mathrm{CV}$ & 72,68 & 76,69 & 83,68 & 60,37 & 66,87 \\
& $\mathrm{x}$ & 1,46 & 2,41 & 2,68 & 3,83 & 4,62 \\
$\mathrm{G} 2$ & $\mathrm{~S}$ & 1,76 & 2,17 & 2,61 & 2,97 & 1,78 \\
& $\mathrm{CV}$ & 119,87 & 90,27 & 97,38 & 77,63 & 37,37 \\
& & & & & & \\
$\mathrm{G} 3$ & $\mathrm{x}$ & 1,66 & 1,80 & 2,02 & 2,14 & 2,56 \\
& $\mathrm{~S}$ & 1,40 & 1,67 & 1,58 & 1,52 & 2,53 \\
& $\mathrm{CV}$ & 84,20 & 92,89 & 78,17 & 70,96 & 98,72 \\
\hline
\end{tabular}
de G1 não mostraram médias estatisticamente divergentes das do G2, embora tenha sido notada tendência à significância, como pode ser visto na tabela 2 e figura 2.

Tem-se empregado a adrenalina como agente indutor da arritmia em um grande número de experimentos, e quase invariavelmente na tentativa de se avaliar a antiarritmogenicidade de fármacos utilizados no pré-tratamento à anestesia volátil (LEMKE $\boldsymbol{e t}$ al., 1994; NIIYA, 1990) e mais raramente, no estudo de fármacos injetáveis.

Neste experimento, foi possível notar que, tanto a quetamina, como a levomepromazina são dotadas de propriedades antiarritmogênicas, não se considerando arritmia, a taquicardia produzida pelo anestésico, embora o bloqueio da atividade da adrenalina tenha sido notadamente superior no G3. Deste modo, permite-se discordar das observaç̃es de BEDNARSKI et al. (1988) já que a ação antiarrítmica da quetamina citada por NIIYA (1990), pôde ser

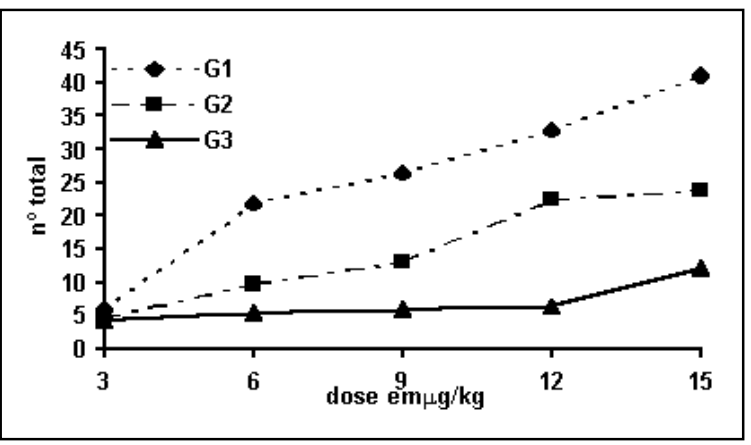

Figura 2 - Variação dos valores médios do número total de batimentos cardíacos de origem não sinusal (ESV), em cães não tratados (G1) ou tratados com quetamina (G2 e G3) e pré-tratados com levomepromazina (G3), submetidos a doses crescentes de adrenalina. 
ventriculares. A este efeito pode imputar-se a competição entre a levomepromazina e a adrenalina pelos receptores tipo $\alpha$ do miocárdio, com vantagem para o primeiro, em detrimento do segundo fármaco. A quantidade de ESV obtida foi inferior àquela observada nos animais não anestesiados. Admite-se, portanto, inferir que agentes $\alpha$-bloqueadores podem colaborar com o sucesso da anestesia, quando há suspeita do desenvolvimento de arritmias por descarga endógena excessiva de catecolaminas ou quando a condição do paciente exige a administração de adrenalina.

\section{CONCLUSÕES}

Com base nos achados obtidos com a metodologia concebida, foi possível admitir, como hipótese conclusiva, que a levomepromazina é indicada na associação com a quetamina e inibe o aparecimento de complexos ectópicos ventriculares, induzidos pela adrenalina, bem como diminui o período de ação da catecolamina.

\section{AGRADECIMENTOS}

Os autores agradecem ao Prof. Dr. Paulo Roberto Curi pelo auxílio na avaliação estatística e à Disciplina de Anestesiologia Veterinária do Depto. de Cirurgia Veterinária e Reprodução Animal da FMVZ/Unesp, Campus de Botucatu pela concessão de prioridade no uso das instalações do Laboratório de Anestesiologia Experimental. Por razões humanitárias não foi adotada metodologia causadora de sofrimento e os animais não foram submetidos à eutanásia.

\section{FONTES DE AQUISIÇÃO}

${ }^{a}$ Cloridrato de adrenalina 1/1000 - Laboratório Santista Ltda. ${ }^{\mathrm{b}}$ Cardioscópio/Oxicapnógrafo mod. DX7100 - Dixtal Ind. \& Com. Ltda.

${ }^{c}$ Eletrocardiógrafo mod. ECG-5 - Funbec.

${ }^{\mathrm{d}}$ Francotar - Indústrias Químicas Lorena Ltda.

${ }^{\text {e}}$ Bomba de Infusão mod. FARS 500 - Lifemed Pesquisas Médicas Ind. e Com. Ltda.

${ }^{f}$ Neozine - Rhodia S. A. Divisão Farmacêutica.

\section{REFERÊNCIAS BIBLIOGRÁFICAS}

BEDNARSKI, R.M., SAMS, R.A., MAJORS, L.J. Reduction of the ventricular arrhytmogenic dose of epinephrine by ketamine administration in halothane-anesthetized cats. Am J Vet Res, v. 46 , p. $350-354,1988$.

CURI, P.R. Análise de medidas repetidas em experimentos biológicos. Rev Bras Estat, v. 41, n. 161, p. 137-150. 1980.

HOFFMAN, B.B., LEFKOWITZ, R.J., Catecolaminas e drogas simpatomiméticas. In: GILMAN, A.G., RALL, T.W., NIES, A.S., TAYLOR, P. Goodman \& Gilman: as bases farmacológicas da terapêutica. 8. ed. Rio de Janeiro: Guanabara, 1991. p. 123-143.

LEMKE, K.A., TRANQUILLI, W.J., THURMON, J.C., $\boldsymbol{e}$ t al., Influence of cholinergic blockade on the development of epinephrine-induced ventricular arrythmias in halothane and isoflurane-anesthetized dogs. Vet Anesth, v. 23, p. 61-66, 1994.

MASSONE, F. Anestesiologia veterinária. Farmacologia e técnicas, 2. ed. Rio de Janeiro: Guanabara, 1994. 235 p.

MORRISON, D.F. Multivariate Statistical Methods. New York: MaC Grows Hill Book, 1967. 388 p.

NIIYA, S. The effect of ketamine on epinephrine-induced arrythmias in dogs anesthetized with halothane-nitrous oxide. Masui, v. 39, n. 12, p. 1652-1659, 1990.

NUNES, N., POMPERMAYER, L.G., PIROLO, J., et al. Emprego do metaraminol no bloqueio da hipotensão produzida pela levomepromazina em cães. Braz J Vet Res An Sci, v. 32, n. 3, p. 120-124, 1995.

PENDER, J.W. Dissociative anesthesia. J Am Med Assoc, v. 215 , p. $1126-1130,1971$.

SHORT, C.E. Principles \& practice in veterinary anesthesia. Baltimore: Willian \& Wilkins, 1987. 669 p.

TRANQUILLI, W.J., THURMON, J.C., BENSON, G.J., et al. Alteration in the arrythmogenic dose of epinephrine (ADE) following xylazine administration to halothane-anesthetized dogs. J Vet Pharmacol Therap, v. 9, n. 2, p. 198-203, 1986.

WYLLIE, W.D., CHURCHILL-DAVIDSON, H.C. Anestesiologia. 3. ed. Rio de Janeiro: Guanabara, 1974. 1031 $\mathrm{p}$

Ciência Rural, v. 29, n. 2, 1999. 